\title{
Towards a kinematic model of the Local Group
}

\author{
Andreas Brunthaler ${ }^{* \dagger}$ \\ Max-Planck Institut für Radioastronomie \\ E-mail: brunthal@mpifr-bonn.mpg.de \\ Mark J. Reid \\ Harvard-Smithsonian Center for Astrophysics \\ E-mail: reidecfa.harvard.edu \\ Heino Falcke \\ ASTRON \\ E-mail: falckelastron.nl \\ Christian Henkel \\ Max-Planck Institut für Radioastronomie \\ E-mail: p220henempifr-bonn.mpg.de
}

Karl M. Menten

Max-Planck Institut für Radioastronomie

E-mail: kmenten@mpifr-bonn.mpg.de

Measuring the proper motions and geometric distances of galaxies within the Local Group is very important for our understanding of its history, present state and future. Currently, proper motion measurements using optical methods are limited only to the closest companions of the Milky Way. However, given that VLBI provides the best angular resolution in astronomy and phasereferencing techniques yield astrometric accuracies of $\approx 10$ micro-arcseconds, measurements of proper motions and angular rotation rates of galaxies out to a distance of $\sim 1 \mathrm{Mpc}$ are feasible. This article presents results of VLBI observations of regions of $\mathrm{H}_{2} \mathrm{O}$ maser activity in the Local Group galaxies M33 and IC 10. Two masing regions in M33 are on opposite sides of the galaxy. This allows a comparison of the angular rotation rate (as measured by the VLBI observations) with the known inclination and rotation speed of the $\mathrm{HI}$ gas disk leading to a determination of a geometric distance of $730 \pm 100 \pm 135 \mathrm{kpc}$. The first error indicates the statistical error of the proper-motion measurements, while the second error is the systematic error of the rotation model. Within the errors, this distance is consistent with the most recent Cepheid distance to M33. Since all position measurements were made relative to an extragalactic background source, the proper motion of M33 has also been measured. This provides a three dimensional velocity vector of M33, showing that this galaxy is moving with a velocity of $190 \pm 59 \mathrm{~km} \mathrm{~s}^{-1}$ relative to the Milky Way. For IC 10, we obtain a motion of $215 \pm 42 \mathrm{~km} \mathrm{~s}^{-1}$ relative to the Milky Way. These measurements promise a new handle on dynamical models for the Local Group and the mass and dark matter halo of Andromeda and the Milky Way.

8th European VLBI Network Symposium

September 26-29, 2006

Toruń, Poland 


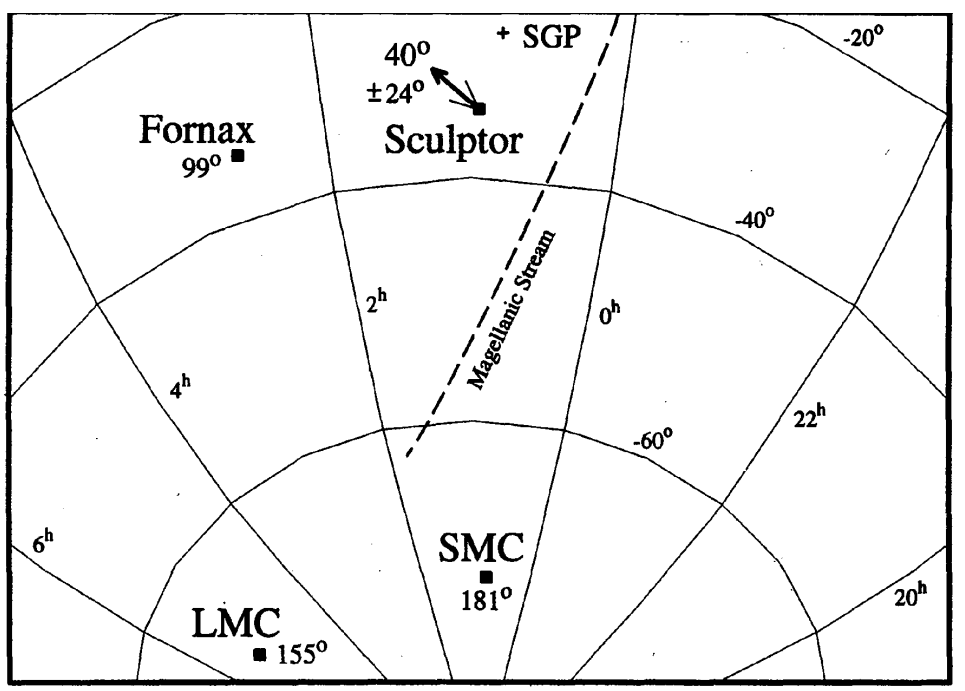

Figure 1: The proper motion of the Sculptor Dwarf Spheroidal Galaxy. Taken from [6].

\section{Introduction}

The nature of spiral nebulae like M33 was debated in the 1920's. While some astronomers favoured a short distance and Galactic origin, others were convinced of its extragalactic nature. In 1923, van Maanen claimed to have measured a large proper motion and angular rotation of M33 from photographic plates separated by $\approx 12$ years [1]. These measurements yielded rotational motions of $\approx 10-30$ mas $\mathrm{yr}^{-1}$, clearly indicating a close distance to M33. However, a few years later, Hubble discovered Cepheids in M33, providing evidence for a large distance to M33 and confirming that M33 is indeed an extragalactic object [2]. The expected proper motions from the rotation of $\mathrm{M} 33$ are then only $\approx 30 \mu$ as $\mathrm{yr}^{-1}, 3$ orders of magnitude smaller than the motions claimed by van Maanen. ${ }^{1}$ After more than 80 years, the idea behind the experiment to measure the rotation and proper motions of galaxies remains interesting for our understanding of the dynamics and geometry of the Local Group. Hence, they are an important science goal of future astrometric missions (e.g. Gaia [3]).

The problem when trying to derive the gravitational potential of the Local Group is that usually only radial velocities are known and hence statistical approaches have to be used. Kulessa and Lynden-Bell introduced a maximum-likelihood method that requires only the line-of-sight velocities, but it is also based on some assumptions (eccentricities, equipartition) [ [

Clearly, the most reliable way of deriving masses is using orbits, which requires the knowledge of three-dimensional velocity vectors obtained from measurements of proper motions. However, measuring proper motions of members of the Local Group to determine its mass is difficult. For the

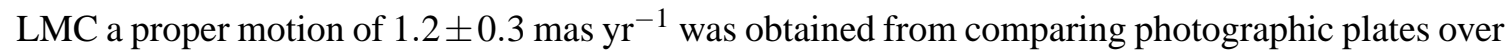
a time-span of 14 years [5]. The Sculptor dwarf spheroidal galaxy moves with $0.56 \pm 0.25 \mathrm{mas} \mathrm{yr}^{-1}$

\footnotetext{
* Speaker.

${ }^{\dagger}$ This research was supported by the DFG Priority Programme 1177.

${ }^{1}$ The source of error in van Maanen's motion determinations was never identified.
} 
obtained from plates spanning 50 years in time (Fig 1], [6]). It was shown that the inclusion of these marginal proper motions can already significantly improve the estimate for the mass of the Milky Way, since it reduces the strong ambiguity caused by Leo I, which can be treated as either bound or unbound to the Milky Way [7].

In recent years, the proper motions of a number of Galactic satellites were measured using the HST [8, 9, 10, 11, 12, 13, 14, 15, 16,. These galaxies are all closer than $150 \mathrm{kpc}$ and show motions between 0.2 and a few milliarcseconds (mas) per year. More distant galaxies, such as galaxies in the Andromeda subgroup at distances of $\sim 800 \mathrm{kpc}$, have smaller angular motions, which are currently not measurable with optical telescopes.

\section{Proper motions with VLBI}

On the other hand, the expected proper motions for galaxies within the Local Group, ranging

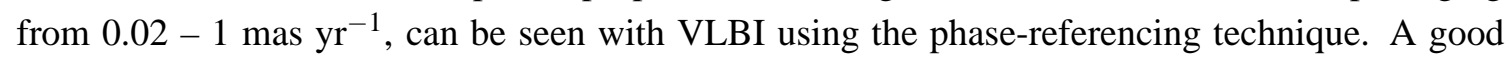
reference point is the motion of Sgr A* across the sky reflecting the Sun's rotation around the Galactic Center. With the VLBA, this motion can be well detected between epochs separated by only one month [17]. New observations increased the time span to 8 years [18]. The position of Sgr A* relative to the background quasar J1745-283 is shown in Figure 目.

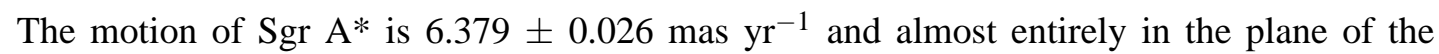
Galaxy. Converted to Galactic coordinates, this gives a proper motion of $-6.379 \pm 0.026 \mathrm{mas} \mathrm{yr}^{-1}$ in Galactic longitude and $-0.202 \pm 0.019 \mathrm{mas} \mathrm{yr}^{-1}$ in Galactic latitude. If one assumes a distance to the Galactic Center $\left(\mathrm{R}_{0}\right)=7.62 \pm 0.32 \mathrm{kpc}$ [19], these motions translate to a velocity of -230 $\pm 10 \mathrm{~km} \mathrm{~s}^{-1}$ along the Galactic plane and $-7.6 \pm 0.6 \mathrm{~km} \mathrm{~s}^{-1}$ out of the plane of the Galaxy. This motion can be entirely explained by a combination of a circular rotation of the Local Standard of Rest (LSR) $\Theta_{0}$ and the deviation of the motion of the Sun from the motion of the LSR. Removing the Solar motion relative to the LSR as measured by Hipparcos data in [20] $\left(5.25 \pm 0.62 \mathrm{~km} \mathrm{~s}^{-1}\right.$ in longitude and $-7.17 \pm \mathrm{km} \mathrm{s}^{-1}$ in latitude) yields an estimate of $\Theta_{0}=225 \pm 10 \mathrm{~km} \mathrm{~s}^{-1}$. Here, the error is dominated by the uncertainty in the distance to the Galactic Center, $\mathrm{R}_{0}$. The motion of Sgr $\mathrm{A}^{*}$ out of the plane of the Galaxy is only $-0.4 \pm 0.8 \mathrm{~km} \mathrm{~s}^{-1}$. This lower limit can be used to put tight constraints on the mass of Sgr A* (for details see [18]).

With the accuracy obtainable with VLBI, one can in principle measure proper motions for most Local Group members very accurately within less than a decade. The main problem so far is finding appropriate radio sources. Useful sources would be either compact radio cores or strong maser lines associated with star forming regions. Fortunately, in a few galaxies, bright masers are already known.

The most suitable candidates for such a VLBI phase-referencing experiment are the strong $\mathrm{H}_{2} \mathrm{O}$ masers in IC $10\left(\sim 10 \mathrm{Jy}\right.$ peak flux in $0.5 \mathrm{~km} \mathrm{~s}^{-1}$ line) and IC 133 in M33 ( 2 Jy, the first extragalactic maser discovered) [21]. The two galaxies belong to the brightest members of the Local Group and are thought to be associated with M31. In both cases, a relatively bright phasereferencing source is known to exist within a degree. In addition, their galactic rotation is well known from H I observations. Consequently, M33 and IC 10 seem to be the best-known targets for attempting to measure Local Group proper motions with the VLBA. 


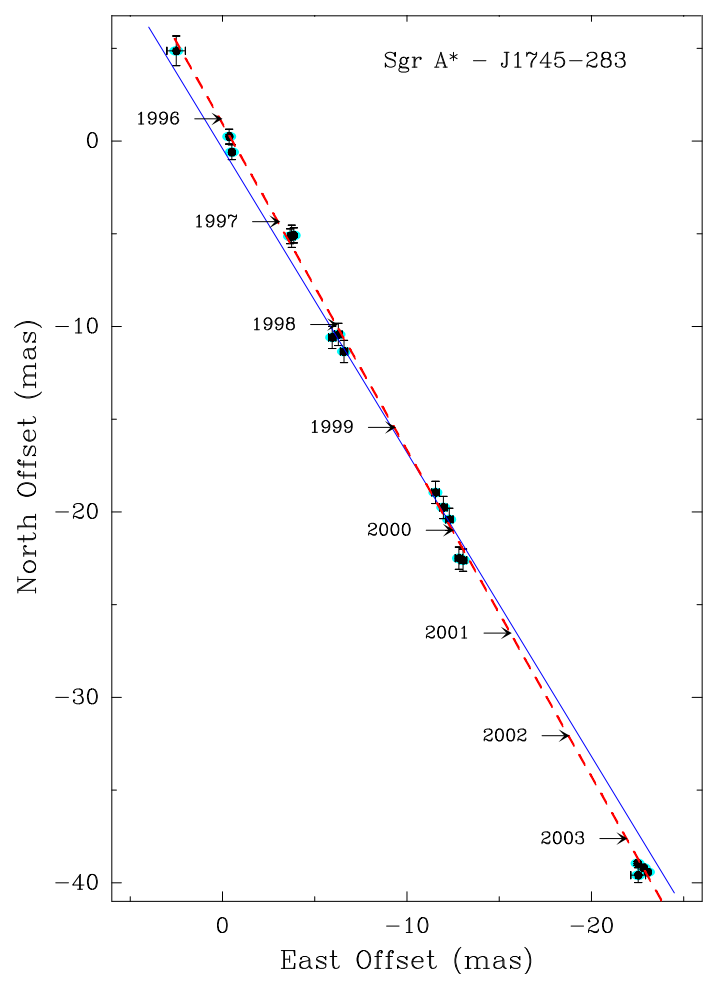

Figure 2: Apparent motion of Sgr A* relative to a background quasar. The blue line indicates the orientation of the Galactic plane and the dashed line is the variance-weighted best-fit proper motion. Taken from [18].

\section{VLBI observations of M33 and IC 10}

\subsection{Observations and data reduction}

We observed two regions of $\mathrm{H}_{2} \mathrm{O}$ maser activity in $\mathrm{M} 33$ (M33/19 and IC 133) eight times with the NRAO VLBA between March 2001 and June 2005 [22]. M33/19 is located in the south-eastern part of M33, while IC 133 is located in the north-east of M33 (see Fig. 3). We observed the usually brightest maser in IC 10-SE with the VLBA thirteen times between February 2001 and June 2005 [28].

The observations involved rapid switching between the phase calibrator and the target sources. With source changes every 30 seconds, an integration time of 22 seconds per scan was achieved. From the second epoch on, we included geodetic-like observations where we observed for $45 \mathrm{~min}$ utes 10-15 strong radio sources ( $>200 \mathrm{mJy})$ with accurate positions $(<1 \mathrm{mas})$ at different elevations to estimate an atmospheric zenith delay error in the VLBA calibrator model (see [18, 23] for a discussion). In the second and third epoch, we used two blocks of these geodetic observations before and after the phase-referencing observations. From the fourth epoch on, we included a third geodetic block in the middle of the observation. The data were edited and calibrated using standard techniques in AIPS, and zenith delay corrections were applied to improve the accuracy of the phase-referencing. The masers in IC 133, M33/19, and IC 10 were imaged with standard techniques in AIPS. 


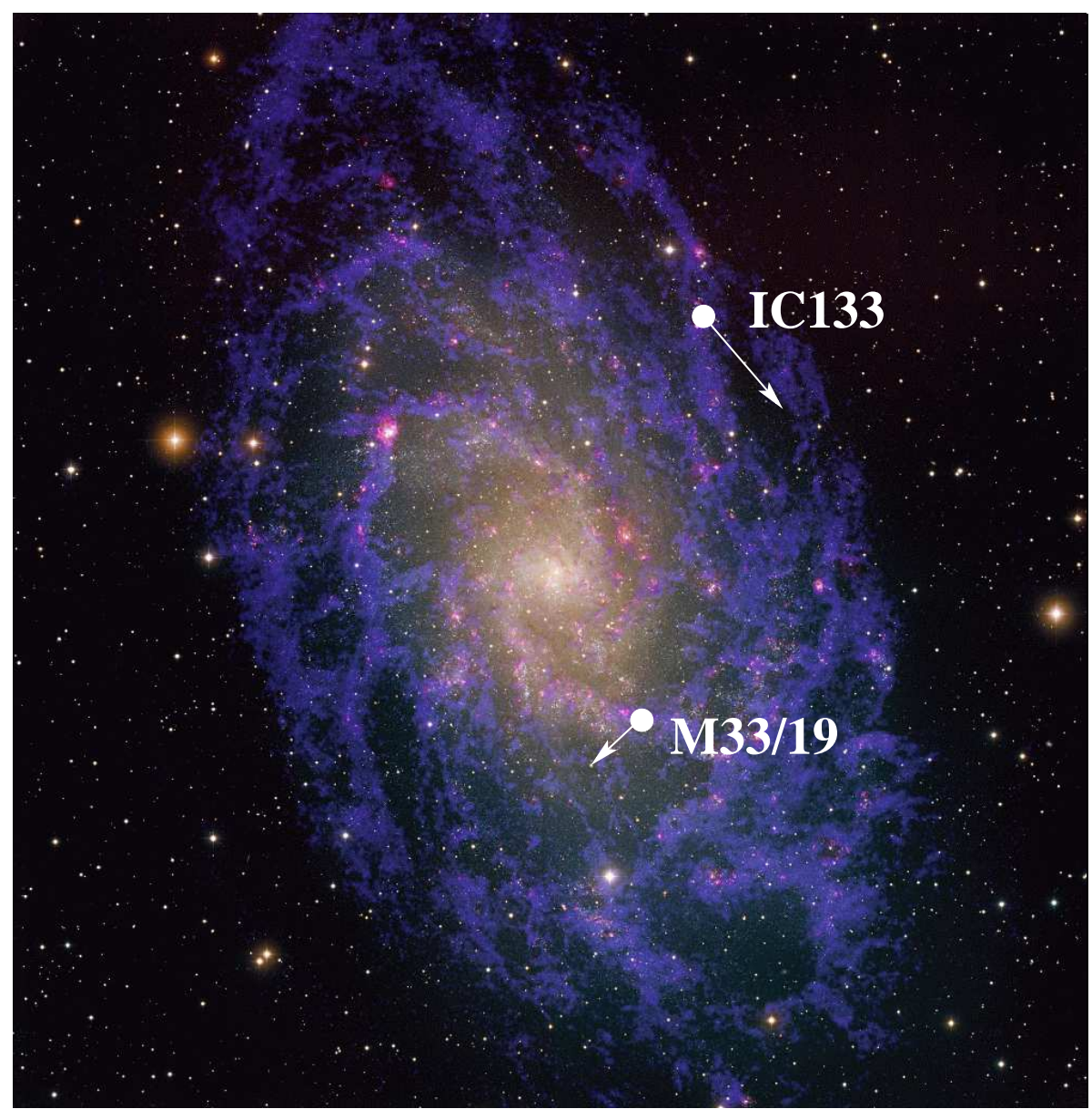

Figure 3: Position of two regions of maser activity in M33. Also shown are predicted motions due to rotation of the H I disk (shown in blue). The optical image of M33 was provided by Travis Rector (NRAO/AUI/NSF and NOAO/AURA/NSF), and the HI image by David Thilker (NRAO/AUI/NSF) and Robert Braun (ASTRON). Taken from [22].

\subsection{Proper motions of M33/19 and IC 133}

The maser emission in M33/19 and IC 133 is variable on timescales of less than one year. Between the epochs, new maser features appeared while others disappeared. However, the motions of four components in M33/19 and six components in IC 133 could be followed over all four epochs. The component identification was based on the positions and radial velocities of the maser emission. Each component was usually detected in several frequency channels. A rectilinear motion was fit to each maser feature in each velocity channel separately. Fits with a reduced $\chi^{2}$ larger than 3 were discarded as they are likely affected by blending. Then, the variance-weighted average of all motions was calculated. This yields an average motion of the maser components in M33/19

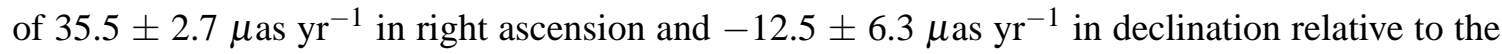

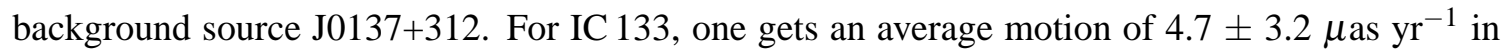

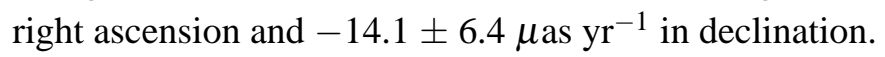



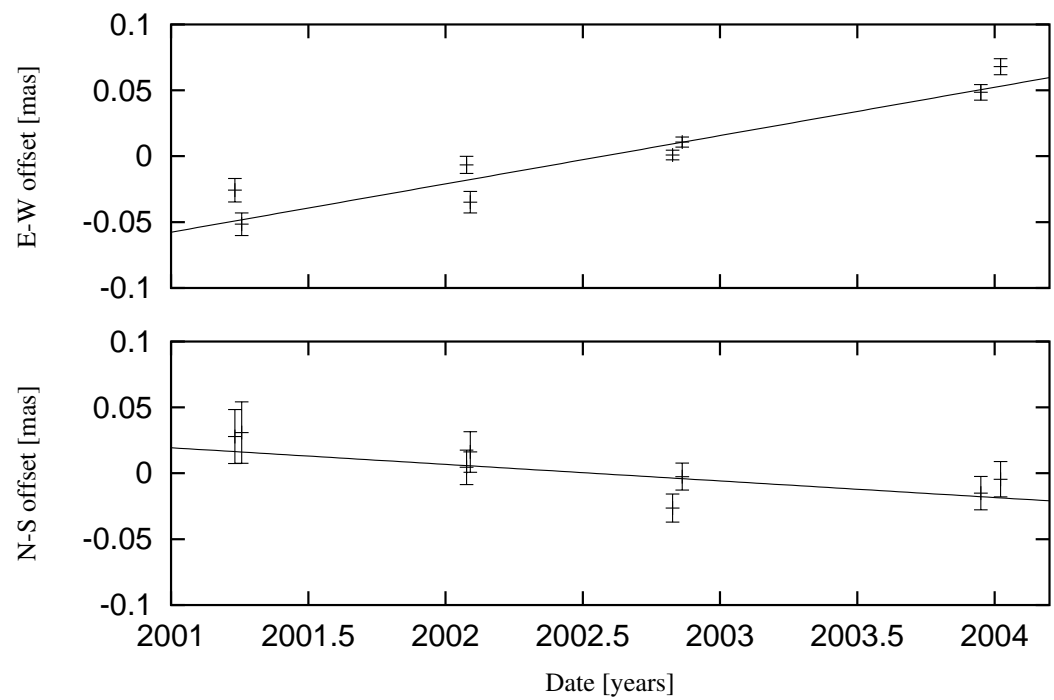

Figure 4: Average positions of the maser in M33/19 in East-West (upper) and North-South (lower) relative to the reference source. Taken from [22].
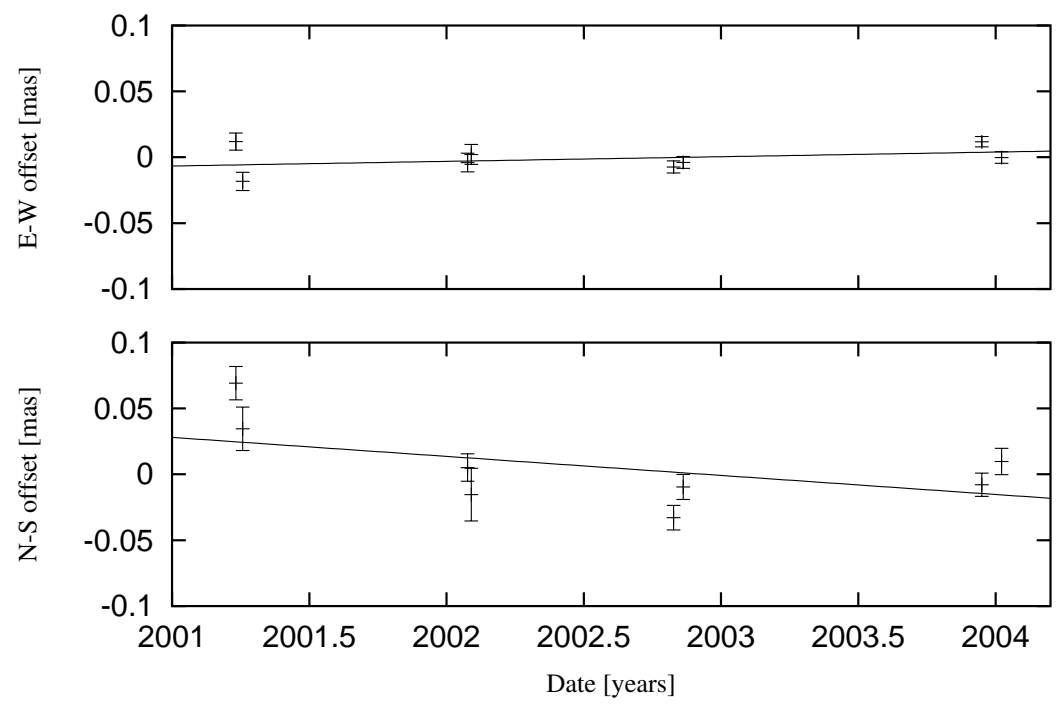

Figure 5: Average positions of the maser in IC 133 in East-West (upper) and North-South (lower) relative to the reference source. Taken from [22].

\subsection{Geometric distance of M33}

The relative motions between M33/19 and IC 133 are independent of the proper motion of M33 and any contribution from the motion of the Sun. Since the rotation curve and inclination of the galaxy disk are known, one can predict the expected relative angular motion of the two masing regions. The rotation of the HI gas in M33 has been measured [24] and one can calculate the expected transverse velocities of M33/19 and IC 133. This gives a relative motion of $106.4 \mathrm{~km} \mathrm{~s}^{-1}$ in right ascension and $35 \mathrm{~km} \mathrm{~s}^{-1}$ in declination between the two regions of maser activity.

The radial velocities of the $\mathrm{H}_{2} \mathrm{O}$ masers in $\mathrm{M} 33 / 19$ and IC 133 and the proximate $\mathrm{HI}$ gas are 
in very good agreement $\left(<10 \mathrm{~km} \mathrm{~s}^{-1}\right)$. This strongly suggests that the maser sources are rotating with the $\mathrm{HI}$ gas in the galaxy. However, while the agreement between the rotation model presented in [24] and the radial velocity of the H I gas at the position of IC 133 is also very good $\left(<5 \mathrm{~km} \mathrm{~s}^{-1}\right)$, there is a difference of $\sim 15 \mathrm{~km} \mathrm{~s}^{-1}$ at the position of M33/19. Hence, we conservatively assume a systematic error of $20 \mathrm{~km} \mathrm{~s}^{-1}$ in each velocity component for the relative velocity of the two maser

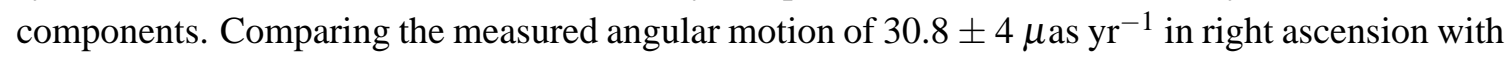
the expected linear motion of $106 \pm 20 \mathrm{~km} \mathrm{~s}^{-1}$, one gets a geometric distance of

$$
D=730 \pm 135 \pm 100 \mathrm{kpc}
$$

where the first error figure indicates the systematic error from the rotation model while the second error is the statistical error from the VLBI proper-motion measurements.

After less than three years of observations, the uncertainty in the distance estimate is already dominated by the uncertainty of the rotation model of M33. However, this can be improved in the near future by determining a better rotation model using higher-resolution (e.g., Very Large Array or Westerbork Synthesis Radio Telescope) data of H I gas in the inner parts of the disk. Also, the precision of the proper-motion measurements will increase with time as $t^{3 / 2}$ for evenly spaced observations.

Within the current errors ,the geometric distance of $730 \pm 100 \pm 135 \mathrm{kpc}$ is in good agreement with recent Cepheid and Tip of the Red Giant Branch (TRGB) distances of $802 \pm 51 \mathrm{kpc}$ [25] and $794 \pm 23 \mathrm{kpc}$ [26], respectively.

\subsection{Proper motion of M33}

The observed proper motion $\tilde{\vec{v}}_{\text {prop }}$ of a maser (M33/19 or IC 133) in M33 can be decomposed into three components $\tilde{\vec{v}}_{\text {prop }}=\vec{v}_{\text {rot }}+\vec{v}_{\odot}+\vec{v}_{M 33}$. Here, $\vec{v}_{\text {rot }}$ is the motion of the masers due to the internal galactic rotation in M33 and $\vec{v}_{\odot}$ is the apparent motion of M33 caused by the rotation of the Sun around the Galactic Center. The last contribution, $\vec{v}_{M 33}$, is the true proper motion of M33 relative to the Galaxy.

Since the motion of the Sun [18, 20] and the rotation of M33 [24] is known, one can calculate the two contributions $\vec{v}_{\text {rot }}$ and $\vec{v}_{\odot}$. Combining these velocity vectors, one gets the true proper motion of M33:

$$
\begin{array}{rc}
\dot{\alpha}_{M 33}= & \dot{\tilde{\alpha}}_{\text {prop }}-\dot{\alpha}_{r o t}-\dot{\alpha}_{\odot} \\
= & -29.3 \pm 7.6 \frac{\mu \mathrm{as}}{\mathrm{yr}}=-101 \pm 35 \frac{\mathrm{km}}{\mathrm{s}} \\
\text { and } & \dot{\tilde{\delta}}_{\text {prop }}-\dot{\delta}_{r o t}-\dot{\delta}_{\odot} \\
\dot{\delta}_{M 33}= & \\
= & 45.2 \pm 9.1 \frac{\mu \mathrm{as}}{\mathrm{yr}}=156 \pm 47 \frac{\mathrm{km}}{\mathrm{s}} .
\end{array}
$$

Finally, the systemic radial velocity of M33 is $-179 \mathrm{~km} \mathrm{~s}^{-1}$ [24]. The radial component of the rotation of the Milky Way toward M33 is $-140 \pm 9 \mathrm{~km} \mathrm{~s}^{-1}$. Hence, M33 is moving with -39 $\pm 9 \mathrm{~km} \mathrm{~s}^{-1}$ toward the Milky Way. This gives now the three-dimensional velocity vector of M33, which is plotted in Fig. 7. The total velocity of M33 relative to the Milky Way is $190 \pm 59 \mathrm{~km} \mathrm{~s}^{-1}$. 
IC10 - NVSS J002108+591132

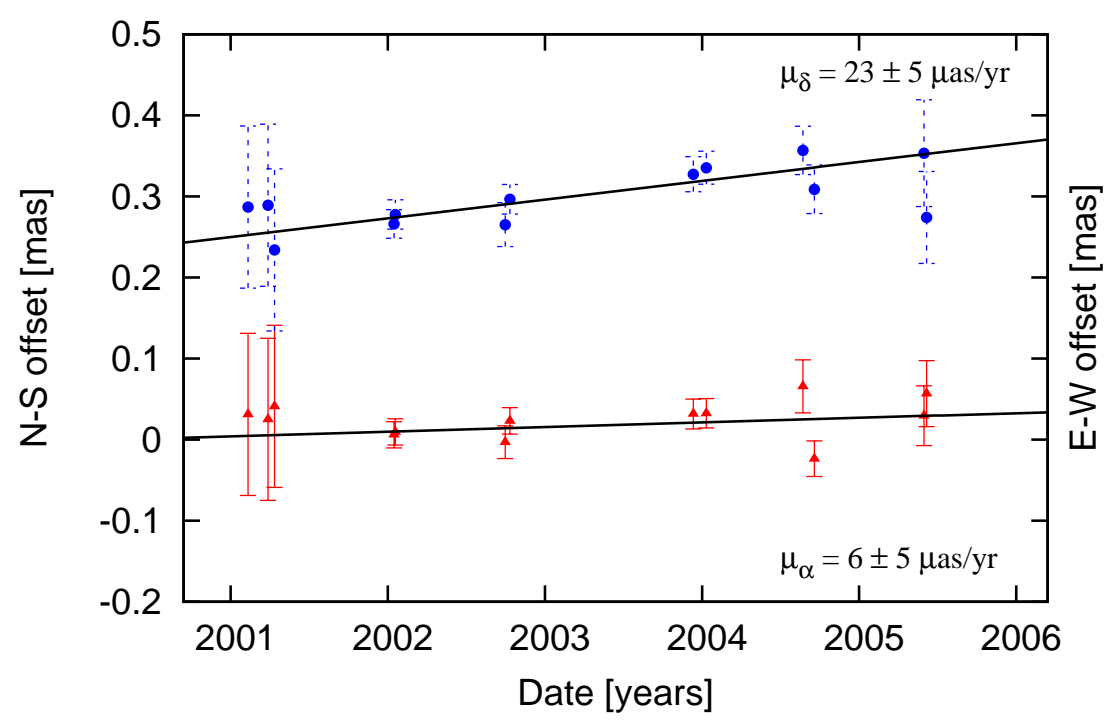

Figure 6: The position of the maser in IC 10 relative to a continuum background source in East-West (red triangles) and North-South (blue circles). The lines show a variance-weighted linear fit to the data points. Taken from [28].

\subsection{Proper motion of IC 10}

In IC 10, only the strongest maser component was detected at all epochs. The position offsets of this maser feature in IC 10 are shown in Fig. 3.5. The uncertainties in the observations of the first epoch are larger than the others, because no geodetic-like observations were made to compensate the zenith delay errors. A rectilinear motion was fit to the data and yielded a value of $6 \pm 5 \mu \mathrm{as} \mathrm{yr}^{-1}$ toward the East and $23 \pm 5 \mu{\text { as } \mathrm{yr}^{-1}}^{-1}$ toward the North.

Once again, the contributions $\vec{v}_{\text {rot }}$ and $\vec{v}_{\odot}$ can be calculated from the known motion of the Sun [18, 20] and the known rotation of IC $10[29,30]$. The true proper motion of IC 10 is then given by:

$$
\begin{aligned}
\dot{\alpha}_{I C 10} & =\dot{\tilde{\alpha}}_{\text {prop }}-\dot{\alpha}_{\text {rot }}-\dot{\alpha}_{\odot} \\
& =-39 \pm 9 \mu \mathrm{as} \mathrm{yr}^{-1}=-122 \pm 31 \mathrm{~km} \mathrm{~s}^{-1} \\
\text { and } & \\
\dot{\delta}_{I C 10} & =\dot{\tilde{\delta}}_{\text {prop }}-\dot{\delta}_{\text {rot }}-\dot{\delta}_{\odot} \\
& =31 \pm 8 \mu \mathrm{as}^{-1}=97 \pm 27 \mathrm{~km} \mathrm{~s}^{-1}
\end{aligned}
$$

The measured systematic heliocentric velocity of IC $10\left(-344 \pm 3 \mathrm{~km} \mathrm{~s}^{-1}\right.$, [31]) is the sum of the radial motion of IC 10 toward the Sun and the component of the solar motion about the Galactic Center toward IC 10, which is $-196 \pm 10 \mathrm{~km} \mathrm{~s}^{-1}$. Hence, IC 10 is moving with $148 \pm 10 \mathrm{~km} \mathrm{~s}^{-1}$ toward the Sun. The proper motion and the radial velocity combined give the three-dimensional 


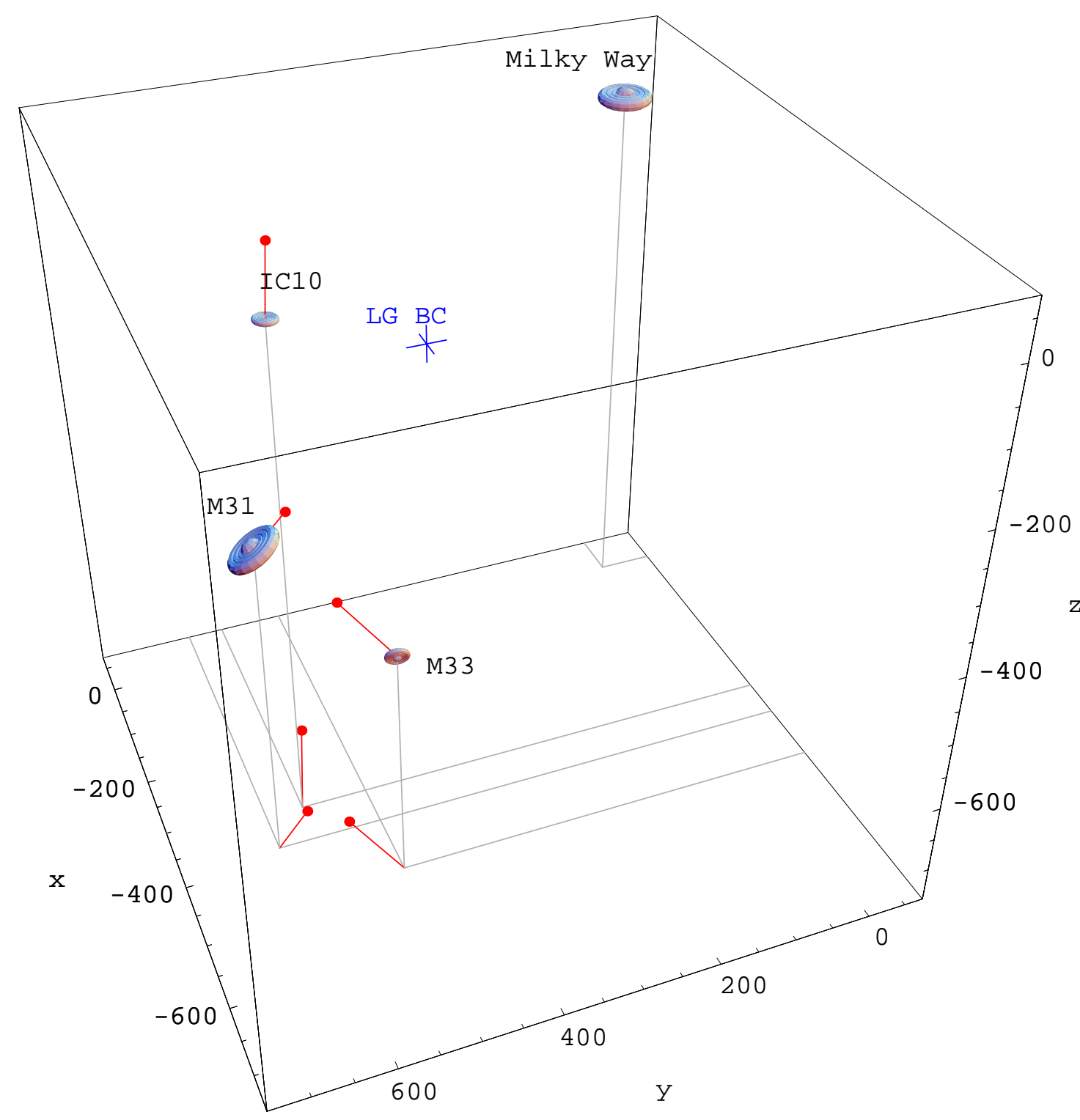

Figure 7: Schematic view of the Local Group with the space velocity of M33 and the radial velocity of Andromeda. The blue cross marks the position of the Local Group Barycenter (LG BC) according to [27]. Taken from [28]. 
space velocity of IC 10. This velocity vector is shown in the schematic view of the Local Group in Fig. 7. The total velocity is $215 \pm 42 \mathrm{~km} \mathrm{~s}^{-1}$ relative to the Milky Way.

\section{Local Group dynamics and mass of M31}

If IC 10 or M33 are bound to M31, then the velocity of the two galaxies relative to M31 must be smaller than the escape velocity and one can deduce a lower limit on the mass of M31:

$$
M_{M 31}>\frac{v_{r e l}^{2} R}{2 G}
$$

A relative velocity of $147 \mathrm{~km} \mathrm{~s}^{-1}$ - for a zero tangential motion of M31 - and a distance of 262 kpc between IC 10 and M31 gives a lower limit of $6.6 \times 10^{11} \mathrm{M}_{\odot}$. One can carry out this calculation for any tangential motion of M31. The results are shown in Fig. 8 (top). The lowest value of 0.7 $\times 10^{11} \mathrm{M}_{\odot}$ is found for a tangential motion of $\mathrm{M} 31$ of $-130 \mathrm{~km} \mathrm{~s}^{-1}$ toward the East and $35 \mathrm{~km} \mathrm{~s}^{-1}$ toward the North.

For a relative motion of $230 \mathrm{~km} \mathrm{~s}^{-1}$ between M33 and M31 - again for a zero tangential motion of M31 - and a distance of $202 \mathrm{kpc}$, one gets a lower limit of $1.2 \times 10^{12} \mathrm{M}_{\odot}$ [22]. Fig. 8 (top) shows also the lower limit of the mass of M31 for different tangential motions of M31 if M33 is bound to M31. The lowest value is $4 \times 10^{11} \mathrm{M}_{\odot}$ for a tangential motion of M31 of $-115 \mathrm{~km} \mathrm{~s}^{-1}$ toward the East and $160 \mathrm{~km} \mathrm{~s}^{-1}$ toward the North.

In [32], it is found that proper motions of M31 in negative right ascension and positive declination would have lead to close interactions between M31 and M33 in the past. These proper motions of M31 can be ruled out, since the stellar disk of M33 does not show any signs of strong interactions.

Thus, we can rule out certain regions in Fig. 8. This results in a lower limit of $7.5 \times 10^{11} \mathrm{M}_{\odot}$ for M31 and agrees with a recent estimate of $12.3_{-6}^{+18} \times 10^{11} \mathrm{M}_{\odot}$ derived from the three-dimensional positions and radial velocities of its satellite galaxies [33].

\section{Summary}

More than 80 years after van Maanen's observation, we have succeeded in measuring the rotation and proper motion of M33 as well as the proper motion of IC 10. These measurements provide a new handle on dynamical models for the Local Group and the mass and dark matter halo of Andromeda and the Milky Way.

We have presented astrometric VLBA observations of the $\mathrm{H}_{2} \mathrm{O}$ masers in the Local Group galaxies M33 and IC 10. We have measured the proper motion of the masers relative to background quasars. Correcting for the internal rotation of M33, IC 10, and the rotation of the Milky Way, these measurements yield proper motions of the two galaxies. The total space velocities relative to the Milky Way of M33 and IC 10 are $190 \pm 59 \mathrm{~km} \mathrm{~s}^{-1}$ and $215 \pm 42 \mathrm{~km} \mathrm{~s}^{-1}$, respectively. If IC 10 and M33 are bound to M31, one can calculate a lower limit of the mass of M31 of $7.5 \times 10^{11} \mathrm{M}_{\odot}$.

Further VLBI observations within the next few years and an improved rotation model have the potential to improve the accuracy of the distance estimate to less than $10 \%$. At least one additional 

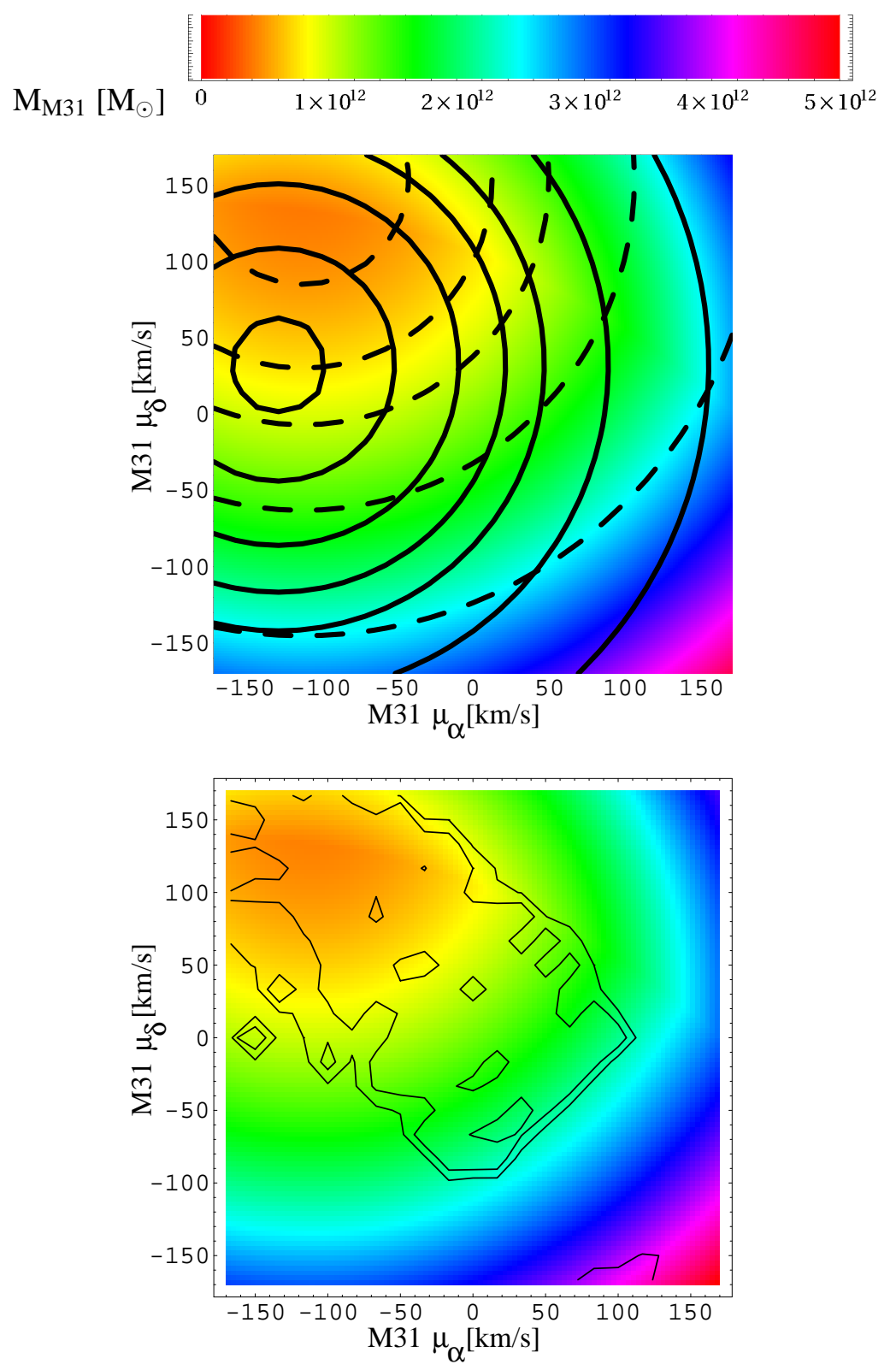

Figure 8: Top: Lower limit on the mass of M31 for different tangential motions of M31 assuming that M33 (dashed) or IC 10 (solid) are bound to M31. The lower limits are $(4,5,7.5,10,15,25) \times 10^{11} \mathrm{M}_{\odot}$ for $\mathrm{M} 33$, and $(0.7,1,2.5,5,7.5,10,15,25) \times 10^{11} \mathrm{M}_{\odot}$ for IC 10, rising from inside. The colour scale indicates the maximum of both values. Bottom: The colour scale is the same as above and gives the lower limit on the mass of M31. The contours show ranges of proper motions that would have lead to a large amount of stars stripped from the disk of M33 through interactions with M31 or the Milky Way in the past. The contours delineate $20 \%$ and $50 \%$ of the total number of stars stripped [32]. These regions can be excluded, since the stellar disk of M33 shows no signs of such interactions. Taken from [28]. 
maser source exists in M33 [21] that will be used in the future to increase the accuracy of the measurements. A third region of maser activity will also help to check for non-circular velocities of the masers.

Unfortunately, no maser emission could be found in Andromeda despite intensive searches. Hence, the proper motion vector of Andromeda is still unknown. In the near future, new technical developments using higher bandwidths will increase the sensitivity of VLBI. This will allow the detection of radio emission from the central black hole of Andromeda, M31*, with flux densities of $\sim 30 \mu \mathrm{Jy}$ [34], to measure the proper motion of Andromeda.

Today, we are only able to study the extreme (bright) tip of the maser luminosity distribution for interstellar masers. The Square Kilometer Array (SKA), with substantial collecting area on intercontinental baselines and a frequency coverage up to $22 \mathrm{GHz}$ [35], will provide the necessary sensitivity to detect and measure the proper motions of a much greater number of masers in active star-forming regions in the Local Group.

\section{References}

[1] A. van Maanen, $A p J$ 1923, 57, 264

[2] E.P. Hubble, ApJ 1926, 63, 236

[3] G.A. Tammann, B. Reindl, Ap\&SS 2002, 280, 165

[4] A.S. Kulessa, D. Lynden-Bell, MNRAS 1992, 255, 105

[5] B.F. Jones, et al., AJ 1994, 107, 1333

[6] A.E. Schweitzer, et al., $A J$ 1995, 110, 2747

[7] C.S. Kochanek, ApJ 1996, 457, 228

[8] S. Piatek, et al., $A J$ 2002, 124, 3198

[9] S. Piatek, et al., $A J$ 2003, 126, 2346

[10] S. Piatek, et al., AJ 2005, 130, 95

[11] S. Piatek, et al., $A J$ 2006, 131, 1445

[12] D.I. Dinescu, et al., $A J$ 2004, 128, 687

[13] D.I. Dinescu, et al., ApJ 2005, 618, 25

[14] D.I. Dinescu, et al., ApJ 2005, 631, 49

[15] N. Kallivayalil, et al., ApJ 2006, 638, 772

[16] N. Kallivayalil, et al., $A p J$ 2006, [astro-ph/0606240]

[17] M.J. Reid, et al., ApJ 1999, 524,816

[18] M.J. Reid, A. Brunthaler, ApJ 2004, 616, 872

[19] F. Eisenhauer, et al., ApJ 2005, 628, 246

[20] W. Dehnen, J.J. Binney, MNRAS 1998, 298, 387

[21] A. Brunthaler, et al., $A \& A$ 2006, 457, 109 
[22] A. Brunthaler, et al., Science 2005, 307, 1440

[23] A. Brunthaler, et al., in Proceedings of "Future Directions in High Resolution Astronomy: The 10th Anniversary of the VLBA", Edited by J. D. Romney \& M. J. Reid. ASP Conference Series, Vol. 340. San Francisco, CA: Astronomical Society of the Pacific, 2005., 455

[24] E. Corbelli, S.E. Schneider, ApJ 1997, 479, 244

[25] M.G. Lee, et al., ApJ 2002, 565, 959

[26] A.W. McConnachie, et al., MNRAS 2005, 356, 979

[27] S. van den Bergh, A\&A Rev. 1999, 9, 273

[28] A. Brunthaler, et al., $A \& A$ 2007, 462, 101

[29] G.S. Shostak, E.D. Skillman, A\&A 1989, 214, 33

[30] E.M. Wilcots, B.W. Miller, AJ 1998, 116, 2363

[31] G. de Vaucouleurs, et al., Third Reference Catalogue of Bright Galaxies. Volume 1-3, XII 1991, Springer-Verlag Berlin Heidelberg New York

[32] A. Loeb, et al., ApJ 2005, 633, 894

[33] N.W. Evans, M.I. Wilkinson, MNRAS 2000, 316, 929

[34] P.C. Crane, J.R. Dickel, J.J. Cowan, ApJ 1992, 390, 9

[35] E. Fomalont, M.J. Reid, New Astronomy Review 2004, 48, 1473 